\title{
Effect of varied shoot/root ratio on growth of maize (Zea mays) under nitrogen-limited conditions: Growth experiment and model calculations
}

\author{
G.R. FINDENEGG \\ Department of Soil Science and Plant Nutrition, Wageningen Agricultural University, P.O. Box 8005, \\ 6700 EC Wageningen, The Netherlands
}

Key words: functional equilibrium, growth model, net assimilation rate, nitrogen nutrition, shoot/root ratio, Zea mays L.

\section{Abstract}

Young maize plants have been grown for two weeks on a perlite/sand mixture under controlled high light conditions at two suboptimal nitrogen levels. The relationships between [1] root dry weight (RDW) and dry weight of the total plants, [2] RDW and total root length (TRL), and [3] TRL and daily $\mathrm{N}$-uptake during the two weeks were different for the two $\mathrm{N}$-levels. In contrast, the relationships between [4] the $\mathrm{N}$-concentrations in shoots (NCS) and in total plants, [5] shoot dry weight and leaf area, and [6] NCS and net assimilation rate (NAR) were similar at both N-levels. Mathematical descriptions of the six experimentally derived relationships were combined in a growth model. Varying relation [1] in the model allowed predictions about the effect of different shoot/root ratio (SRR) on growth. Both experimentally observed SRR-relations were nearly optimal for the respective N-levels. Therefore, the SRR established by the plants was close to the 'functional equilibrium' proposed for root and shoot growth.

\section{Introduction}

Deficiencies of water or nutrients can strongly decrease the shoot/root ratio (SRR) of plants (Brouwer, 1962). In this way growth depression resulting from the limiting factor can be minimized. For example, when nitrogen is deficient, increased root development will lead to an increase in nitrogen uptake, because larger soil volumes can be exploited.

The mechanism of SRR adaptation has been disputed (Van Andel et al., 1983; Wilson, 1988). The classical theory of a 'functional equilibrium' between root and shoot states that under all growth conditions the roots have a priority in the use of the nitrogen taken up whereas the shoot has a similar priority for the products of photosynthesis. Consequently, when nitrogen supply falls short, both the relative shortage of nitrogen and the relative excess of carbohydrates in the plant will affect root development less seriously than shoot development. The envisaged "equilibrium" is stable, i.e. any deviation from optimum SRR will be corrected immediately and the growth rate of the plant will be maintained at its maximum.

Davidson (1969) has formulated that according to this principle

$$
\frac{\text { specific root activity } \times \text { root mass }}{\text { specific shoot activity } \times \text { shoot mass }}=\text { constant }
$$

Since the development of this theory our knowledge about priorities in nitrogen allocation within the plant has been extended considerably (Pate, 1980). Recent investigations do not support the simple equilibrium theory (Lambers, 1983). Rather, the nutrient status of plants may influence the production of plant hormones (Kuiper et al., 1989) that in turn determine the distribution of assimilates (Marschner, 1986) and nitrogen (Simpson et al., 1982). Therefore, the 
self-optimalisation of the SRR cannot be taken as guaranteed any more.

In relation with crop production the question arises therefore, whether the growth of plants under nutrient limited conditions may be improved by a manipulation of SRR (e.g. by application of hormones).

This question is difficult to answer experimentally. Application of hormones may cause other physiological responses besides a shift in SRR. The effect of root pruning or defoliation is only transient, because plants quickly return to their original SRR (Brouwer, 1962). Evaluating formula (1) with experimental data is embarrassed by the fact that usually SRR and the activities of roots and shoots are continuously changing during growth (Cooper and Thornley, 1982; Hunt and Burnett, 1973).

The aim of the present study was to evaluate the effect of different SRR's on growth rate by means of dynamic simulation. Nitrogen uptake rate was chosen as the factor limiting root activity and photosynthetic dry matter increase was considered as the principal shoot activity. Maize plants were grown at two N-levels, both growth limiting, so that different SRR's were established. The net assimilation rate as a function of leaf nitrogen and the nitrogen uptake rate as a function of root length were followed during two weeks. Subsequently the effect of various SRR's could be estimated for both N-levels by means of a dynamic simulation model.

\section{Methods}

Maize seedlings (Zea mays L. LG 11) have been grown in $17 \mathrm{~L}$-containers on a sand/perlite mixture $(1: 2 \mathrm{v} / \mathrm{v})$. Once a day the pots were rinsed with an excess of nutrient solution which replaced the total moisture of the pot. The composition of the basic nutrient solution was: $5 \mathrm{mM}$ $\mathrm{CaCl}_{2}, 2 \mathrm{mM} \mathrm{MgSO}_{4}, 2 \mathrm{mM} \mathrm{KH}_{2} \mathrm{PO}_{4}, 2 \mathrm{~m} M$ $\mathrm{K}_{2} \mathrm{SO}_{4}$, Fe-EDTA and micronutrients.

There were two nitrogen treatments: 15 pots were treated with the basic solution containing $0.2 \mathrm{mMCa}\left(\mathrm{NO}_{3}\right)_{2}$ ('N-level 0.4 '), and 15 pots with solution containing $1.0 \mathrm{mM} \mathrm{Ca}\left(\mathrm{NO}_{3}\right)_{2}$ ('Nlevel 2.0').
Growth conditions were $25^{\circ} \mathrm{C}$, a light-dark cycle of $16: 8$ hours (light intensity 90 Watt $/ \mathrm{m}^{2}$ ), and a relative air humidity of $85 \%$. After one week three replicate pots (with 4 plants each) were harvested from both $\mathrm{N}$-levels (=day 0 ). The following harvests were on day $3,7,10$, and 14 , when three pots with $2,1,1$ and 1 plants were harvested, respectively.

Leaf area was determined by a leaf area meter, root length by the line intersect counting method, dry weight after drying at $70^{\circ} \mathrm{C}$, and total- $\mathrm{N}$ concentrations in destructed root and shoot material with the indophenol blue method (Novozamsky et al., 1974).

\section{Results and discussion}

\section{Basic growth data}

In Figure 1 dry weight and total $\mathbf{N}$ content of roots and shoots, leaf area and root length are plotted for both N-levels against time. The solid lines shown in the figure are polynomial regression lines, calculated using the formula

$$
\ln x(t)=a+b \cdot t+c \cdot t^{2}+d \cdot t^{3}
$$

where $\ln x(t)$ is the logarithm of the parameter $x$ at time $t$ (in days), and $a, b, c$, and $d$ are the polynomial coefficients. The correlation coefficients (r) are indicated in the figure.

\section{Growth relationhips}

Relationships between growth parameters, as deduced from basic growth data were used for the growth model. In this section it is described how these relationships were derived. Regression data of the basic parameters as presented in Figure 1, with daily intervals were used for the calculations. Root dry weight was plotted against total dry weight (Fig. 2). As expected, the SRR at the $\mathrm{N}$-level 2.0 was higher than at the $\mathrm{N}$-level 0.4 . However, SRR was not constant but increased with age at both $\mathrm{N}$-levels. This resulted in positive intercepts of the regression lines on the DMR-axis (Fig. 2). The line linking root dry weight and root length (Fig. 3) is steeper at the 

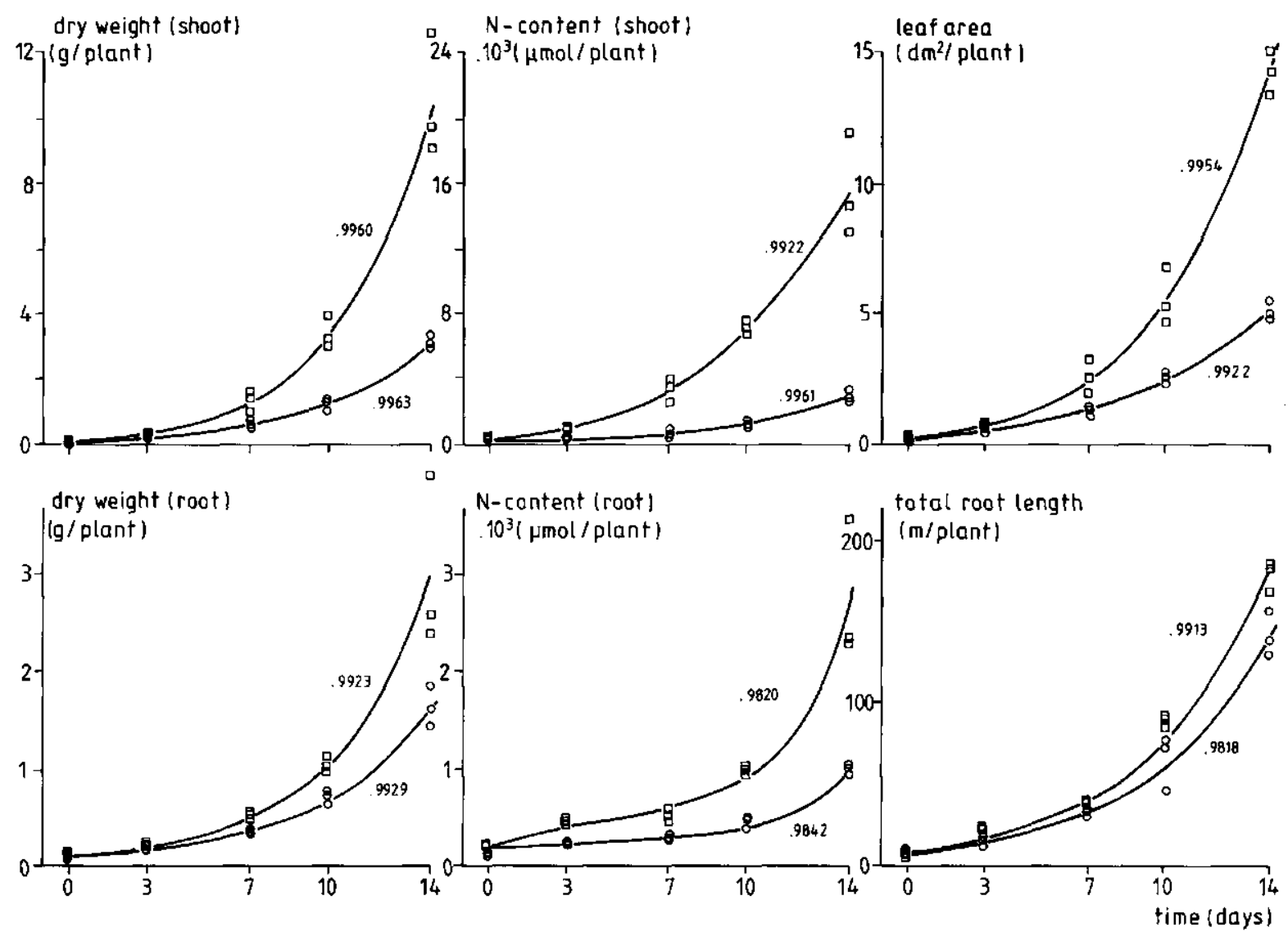

Fig. 1. Dry weight (g/plant) and total N-content ( $\mu \mathrm{mol} / \mathrm{plant})$ of roots and shoots, leaf area (dm ${ }^{2} /$ plant) and total root length (m/plant) of young maize plants during two weeks of growth under controlled conditions. $\mathrm{O}$-level $0.4 ; \square \mathrm{N}-\mathrm{level} 2.0$. Polynomial regression lines; the numbers indicated are correlation coefficients.

0.4 compared to the $2.0 \mathrm{~N}$-level, indicating that thinner roots were formed at the lower $\mathrm{N}$-level.

The daily rinsing of the root medium with nutrient solution prevented depletion of the rooting medium and led to a rather constant daily $\mathrm{N}$-uptake rate per $\mathrm{cm}$ root length $(\Delta \mathrm{NT})$ as seen from the nearly proportional increasing lines in Figure 4. The uptake rate (slope of the line) was roughly 5 times higher for the $\mathrm{N}$-level 2.0 compared to the level 0.4 . This corresponds with the fivefold $\mathrm{NO}_{3}$-concentration in the nutrient solution of this treatment. In Fig. 5 the nitrogen concentration in the shoot (NCS) is plotted as a function of the nitrogen concentration in the total plant (NCT). NCS can fairly well be described as $1.16 \times \mathrm{NCT}$ for both $\mathrm{N}$ levels.

The net assimilation rate (NAR) has been calculated by dividing the daily dry weight in- crease $\triangle \mathrm{DMT}$ by the leaf area. The relation between NCS and the NAR is presented in Figure 6. For the description a modified Michaelis-Menten curve has been used that allows for a minimum concentration in the tissue.

Apparently, the dependence of NAR from NCS is influenced by age. For both N-levels the NAR was overestimated at the beginning of the growth period whereas it was underestimated at the end (solid arrows). There are several explanations for this. For example, the mean light intensity experienced may have increased with plant size, because of the decreasing distance from the light source. Further, the ratio of leaf to stem weight changed during the experiment and the conversion of assimilates into leaf and stem material may occur with different efficiency.

A correction for this age effect has been con- 


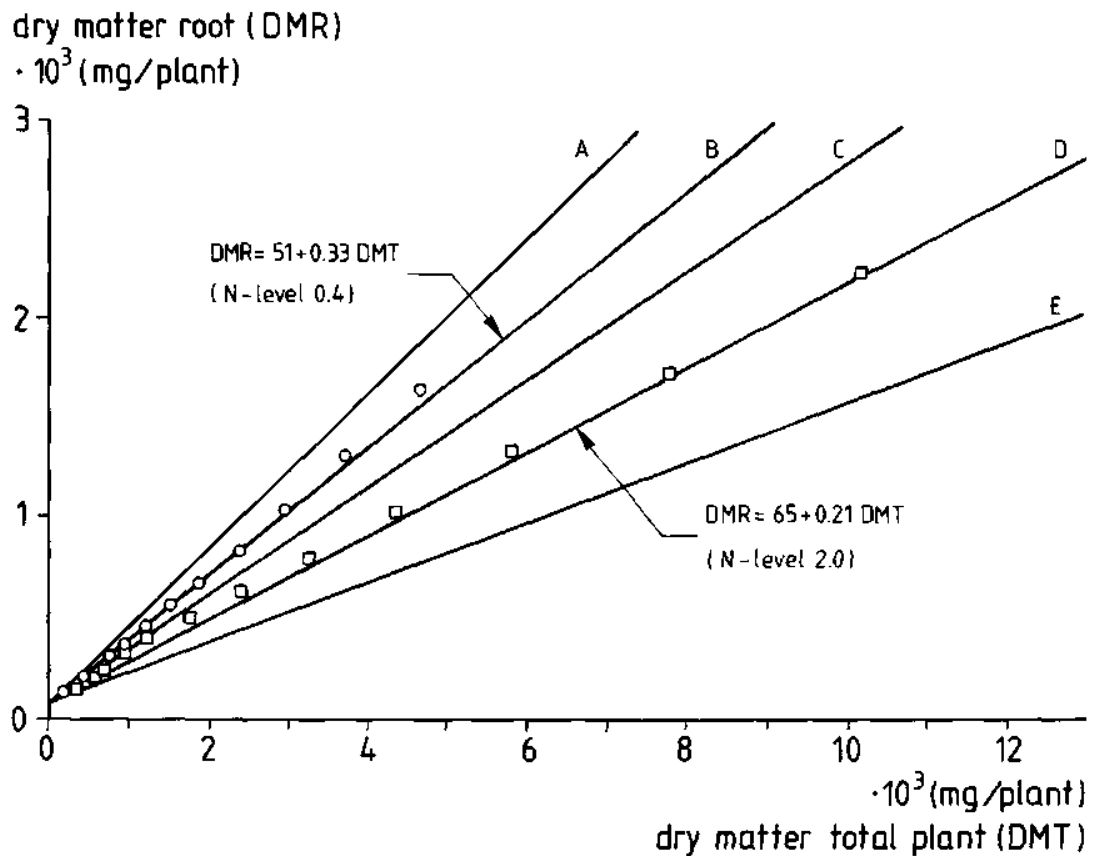

Fig. 2. Relation between dry matter of roots and dry matter of total plants as derived from regression data from Fig. 1. Symbols as in Fig. 1. For lines $\mathrm{A}, \mathrm{C}$ and $\mathrm{E}$ see text.

ducted. This correction was based on a quadratic regression of the relationship between [NAR(exp)/NAR(calc)] and time. Data of both $\mathrm{N}$-levels were pooled. This correction removed $64 \%$ of the deviations of the experimental from the calculated NAR. There was no visible difference in the relationship between dry weight of the shoot and leaf area between the N-levels (Fig. 7), suggesting that there was little effect of nitrogen supply on specific leaf area.

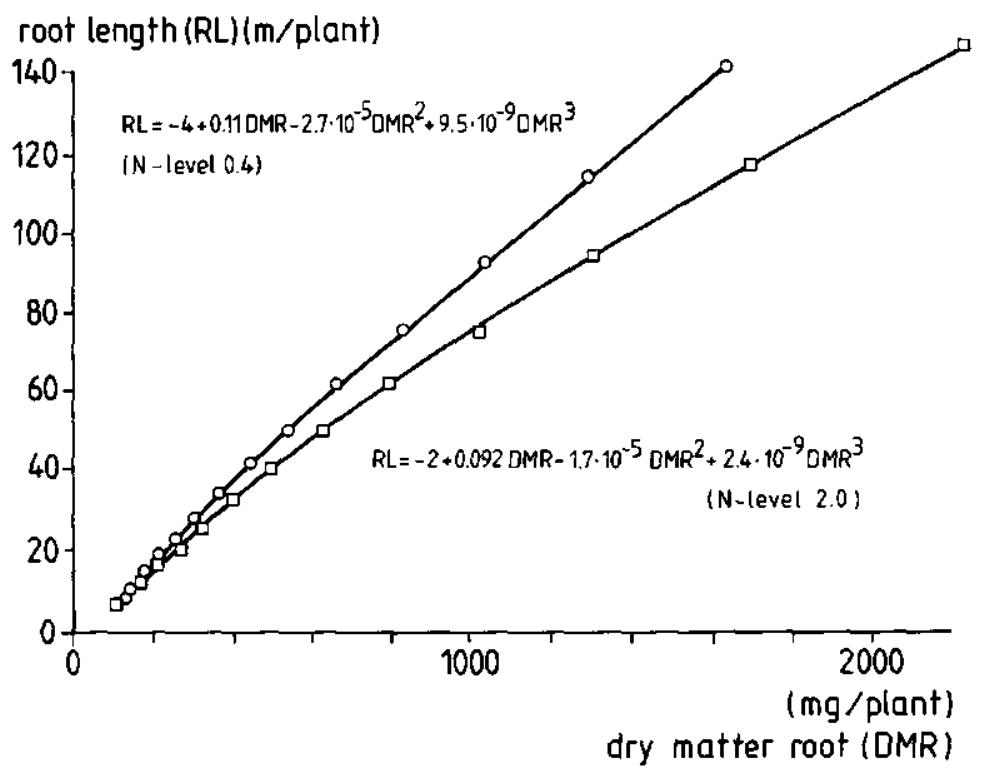

Fig. 3. Relation between the dry matter of roots and total root length as derived from regression data from Fig. 1. Symbols as in Fig. 1. 


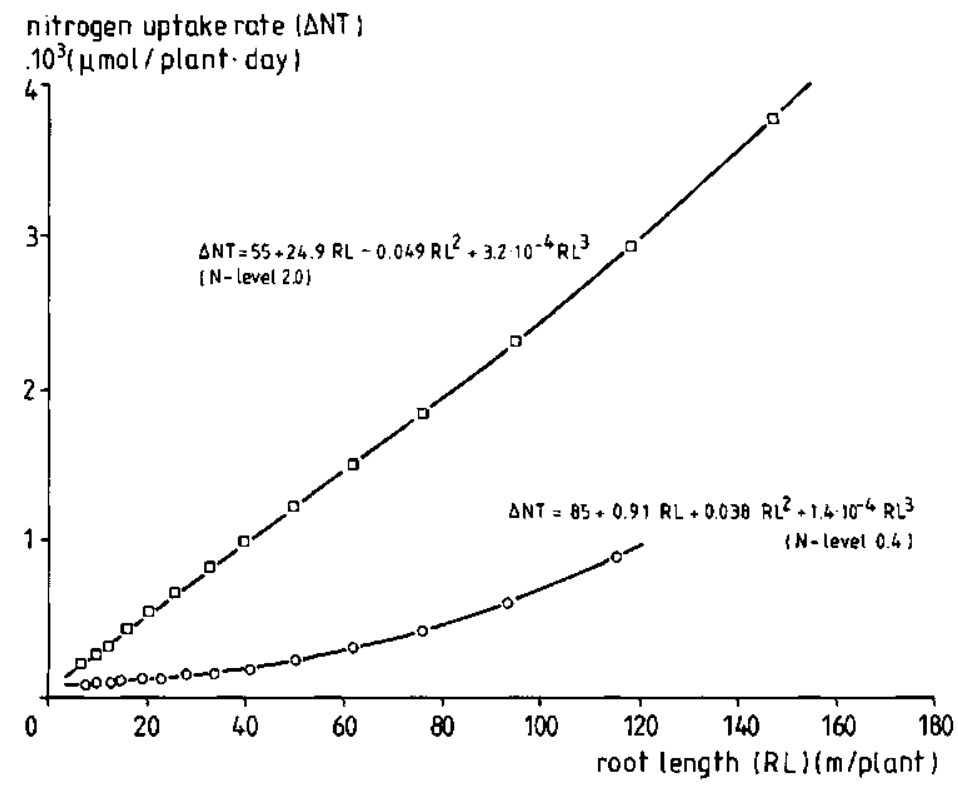

Fig. 4. Relation between daily nitrogen uptake and root length as derived from regression data from Fig. 1. Symbols as in Fig. 1.

\section{Growth model construction}

The model starts with the total dry matter (DMT) and the total amount of nitrogen of one plant (NT) at day 0 . The variation of SRR is introduced into the model by dividing DMT into

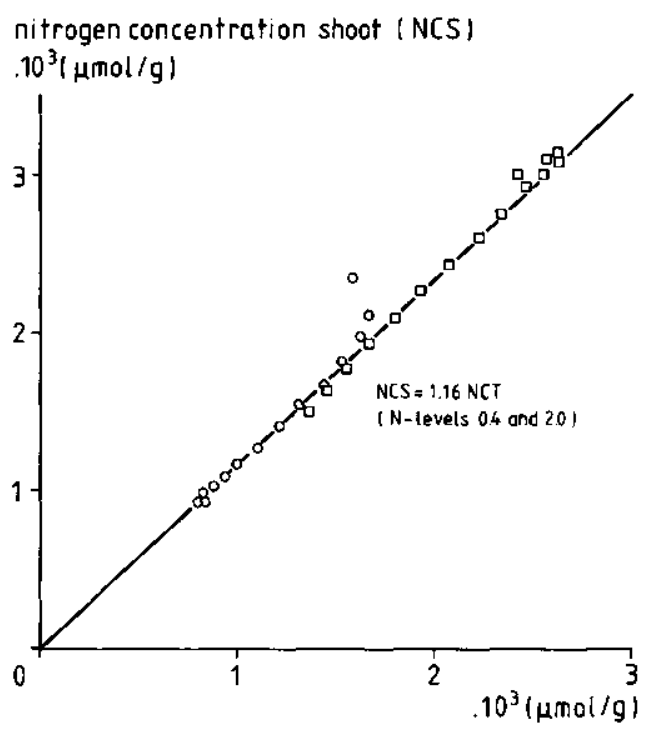

nitrogen concentration total plant (NCT)

Fig. 5. Relation between the nitrogen concentration in the total plant and the nitrogen concentration in the shoots as derived from regression data from Fig. 1. Symbols as in Fig. 1. root and shoot weight (DMR and DMS, resp.) according to the relationships $A$ to $E$ depicted in Figure 2. Besides the relations B (derived from $\mathrm{N}$-level 0.4 ) and $\mathrm{D}(\mathrm{N}$-level 2.0) an intermediate relationship $(\mathrm{C})$ as well as two extreme ones (A and $E$ ) have been used. Subsequently root length $\mathrm{RL}$ is calculated from DMR according to Figure 3 and daily nitrogen uptake $\triangle \mathrm{NT}$ from RL according to Figure 4.

Next the nitrogen concentration of the total plant is calculated by dividing NT by DMT, and the nitrogen concentration in the shoot (NCS) is calculated as a function of NCT (Fig. 5). The net assimilation rate (NAR) is subsequently calculated from NCS (Fig. 6).

Total leaf area (LA) is obtained from DMS according to Figure 7 . Finally the daily dry matter increase $\triangle \mathrm{DMT}$ is calculated as the product of LA and NAR, and DMT and NT are updated by adding $\triangle \mathrm{DMT}$ and $\Delta \mathrm{NT}$, respectively. The cycle is repeated for each day of the experiment.

\section{Results of model calculations}

The model was run with the parameters calculated from plants from both $\mathrm{N}$-levels, using theDMT/DMR relationships $A$ to $E$ in either case. When the "real" DMT/DMR relations have been used the calculated growth data did 


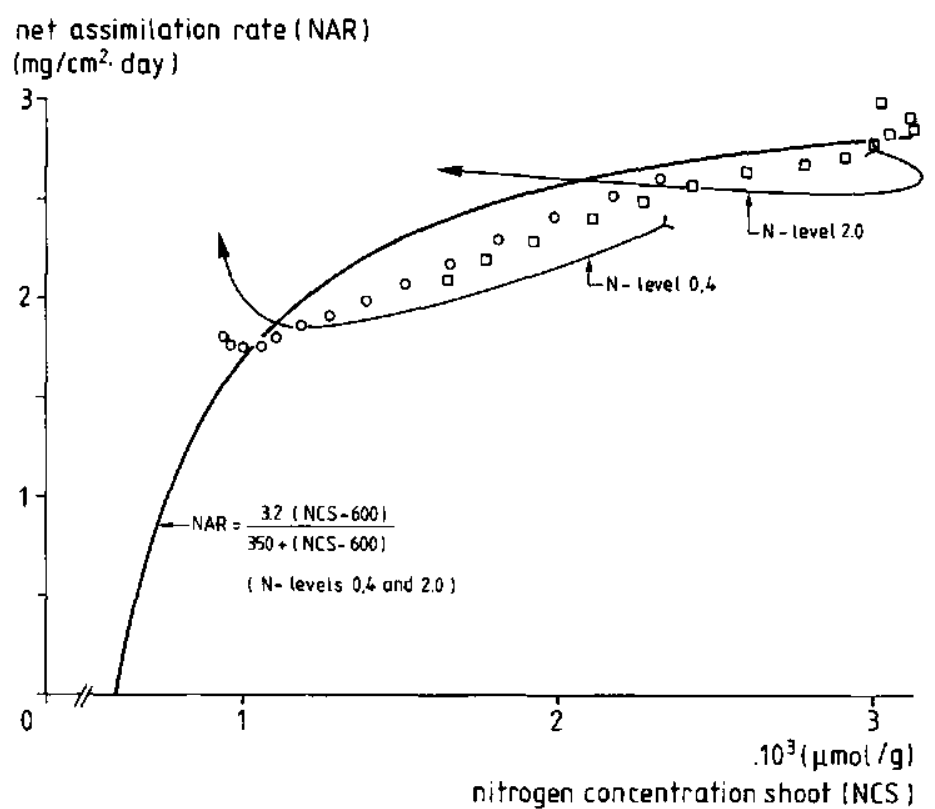

Fig. 6. Relation between the nitrogen concentration in the shoot and the net assimilation rate as derived from regression data from Fig. 1. The relation has been corrected for the time effect (symbols as in Fig. 1) or not corrected (solid arrows).

not deviate too much from the experimental. Typical deviations were $10 \%$; corresponding to the variation between the experimental replicates (Fig. 1). This was true for both nitrogen levels and throughout the experimental period, including final values (Fig. 8). When SRR was varied at the $\mathrm{N}$-level 0.4 , DMT was not affected very much. There was a broad range of SRR where nearly optimal growth was realized. The actual SRR of the N-level 0.4 (relation B) was close to the optimum. At the $\mathrm{N}$-level 2.0 the effect of SRR on final weight was much more prounced but again the actual relation (D) was close to the calculated optimum.

It was tested whether this conclusion was still valid when single coefficients in the model were

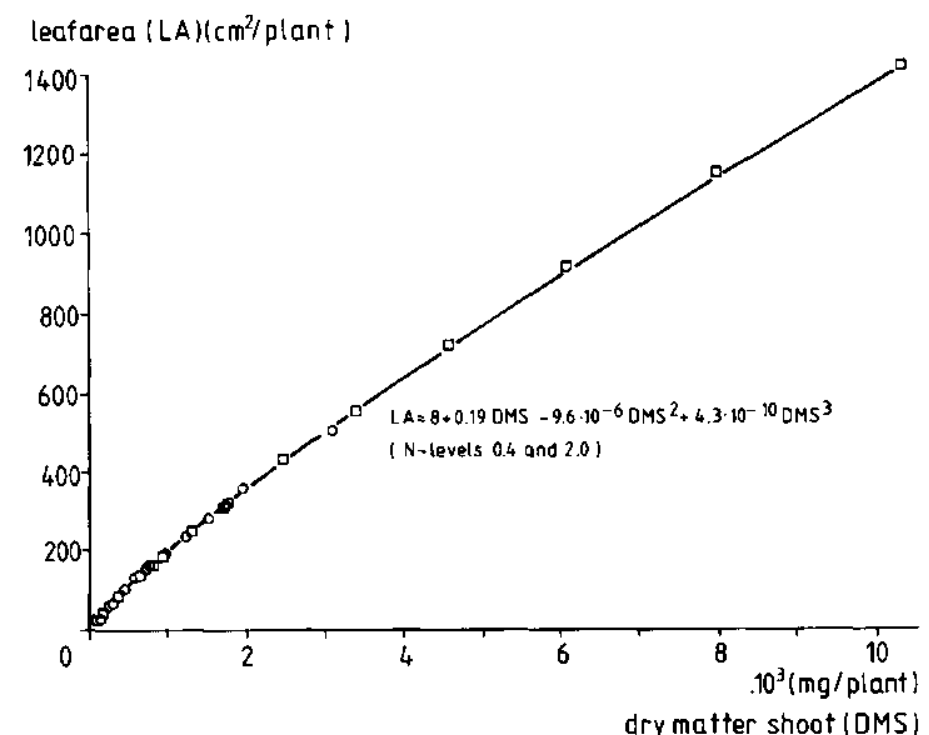

Fig. 7. Relation between the leaf area and the dry matter of shoots as derived from regression data from Fig. 1. Symbols as in Fig. 1. 


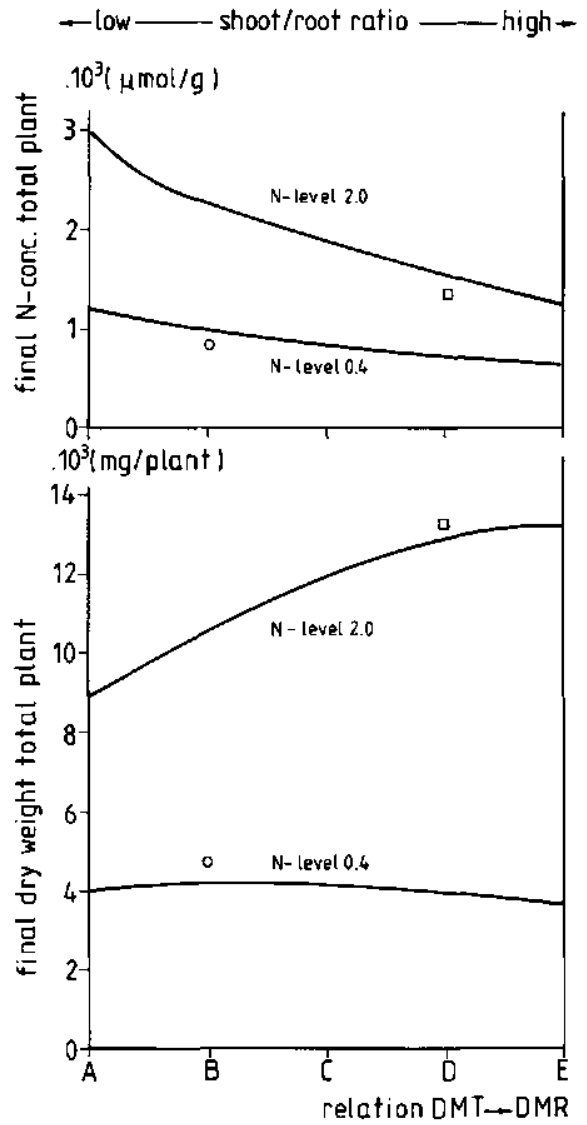

Fig. 8. Solid lines: calculated effect of shoot/root ratio on final dry weight and final $\mathrm{N}$-concentration of plants. Shoot/ root ratio according to relations $A$ to $E$ from Fig. 2. Calculations based on a growth model using the growth relations depicted in Figs. 3 to 7. Symbols (see Fig. 1): experimental regression data.

changed. Generally, the model was not very sensitive to altered coefficients, except for the constants used in the NCS-NAR relationship (Fig. 6). Especially the value of the minimum concentration affected the position of the optimum SRR at the $\mathrm{N}$-level 0.4 . The value used $(600 \mu \mathrm{mol} / \mathrm{g})$, has been measured directly in another experiment where maize plants have ceased to grow following nitrogen exhaustion in the growth medium under similar growth conditions. Therefore this value is reliable and the conclusion safe. It has also been ascertained that the position of the optimum SRR at both $\mathrm{N}$ levels did not change significantly whether the time correction of the NCS-NAR relationship (Fig. 6) has been conducted or not.

Concluding, the model calculations indicate that the SRR realized by the plants was close to the optimum value. Thus, although the SRR established in plants is probably not the result of a 'functional equilibrium' but rather of a hormonal regulation (see Introduction), the realized SRR value does not significantly deviate from the value that would have been established by a 'functional equilibrium' mechanism. One would not expect therefore that a manipulation of the SRR of crops under nitrogen limited conditions will improve their nitrogen efficiency significantly.

\section{References}

Andel O M van, Soekarjo R and Verkaar H J P A (Eds.) 1983 Functional equilibrium between shoots and roots. Neth. J. Agric. Sci. 31, 283-356.

Brouwer R 1962 Nutritive influences on the distribution of dry matter in the plant. Neth. J. Agric. Sci. 10, 399-408.

Cooper A J and Thornley J H M 1976 Response of dry matter partitioning, growth and carbon and nitrogen levels in the tomato plant to changes in root temperature: Experiment and theory. Ann. Bot. 40, 1139-1152.

Davidson R L 1969 Effect of root/leaf temperature differentials on root/shoot ratios in some pasture grasses and clover. Ann. Bot. 33, 561-569.

Hunt R and Burnett J A 1973 The effects of light intensity and external potassium level on root/shoot ratio and rates of potassium uptake in perennial ryegrass (Lolium perenne L.). Ann. Bot. 37, 519-537.

Lambers H 1983 The functional equilibrium, nibbling on the edges of a paradigm. Neth. J. Agric. Sci. 31, 305-311.

Kuiper D, Schuit J and Kuiper P J C 1989 Effects of internal and external cytokinin concentrations on root growth and shoot to root ratio of Plantago major ssp pleiosper$m a$ at different nutrient conditions. In Structural and Functional Aspects of Transport in Roots. Eds. B C Loughman, O Gašpaŕiková and J. Kolek. pp 183-188. Kluwer Academic Publishers, Dordrecht, The Netherlands

Marschner H 1986 Mineral Nutrition of Higher Plants. Academic Press, London. 674 p.

Novozamsky I, Van Eck R, Van Schouwenburg $\mathrm{J}$ Ch and Walinga I 1974 Total nitrogen determination in plant material by means of the indophenol blue method. Neth. J. Agric. Sci. 22, 3-5.

Pate J S 1980 Transport and partitioning of nitrogenous solutes. Annu. Rev. Plant Physiol. 31, 313-340.

Simpson R J, Lambers H and Dalling M J 1982 Kinetin application to roots and its effect on uptake, translocation and distribution of nitrogen in wheat (Triticum aestivum) grown with a split root system. Physiol. Plant. $56,430-435$.

Wilson J B 1988 A review of evidence on the control of shoot: root ratio, in relation to models. Ann. Bot. 61, 433-449. 